\title{
79.
}

\section{ON A SYSTEM OF EQUATIONS CONNECTED WITH MALFATTI'S} PROBLEM, AND ON ANOTHER ALGEBRAICAL SYSTEM.

[From the Cambridge and Dublin Mathematical Journal, vol. Iv. (1849), pp. 270-275.]

CoNSIDER the equations

$$
\begin{aligned}
& b y^{2}+c z^{2}+2 f y z=\theta^{2} a\left(b c-f^{2}\right), \\
& c z^{2}+a x^{2}+2 g z x=\theta^{2} b\left(c a-g^{2}\right), \\
& a x^{2}+b y^{2}+2 h x y=\theta^{2} c\left(a b-h^{2}\right)
\end{aligned}
$$

or, as they may be more conveniently written,

$$
\begin{aligned}
& b y^{2}+c z^{2}+2 f y z=\theta^{2} a \mathfrak{A}, \\
& c z^{2}+a x^{2}+2 g z x=\theta^{2} b \mathfrak{B}, \\
& a x^{2}+b y^{2}+2 h x y=\theta^{2} c \mathfrak{C} .
\end{aligned}
$$

The second and third equations give

$$
\left(g^{2} \mathfrak{C}-h^{2} \mathbb{B}\right) x^{2}-b^{2} \mathbb{B} y^{2}+c^{2} \mathfrak{C} z^{2}+2 c g \mathfrak{C} z x-2 b h \mathfrak{B} x y=0,
$$

hence $\left\{\left(g^{2} \mathfrak{C}-h^{2} \mathfrak{b}\right) x-b h \mathfrak{b} y+c g \mathbb{C} z\right\}^{2}-\mathfrak{B} \mathfrak{C} \mathbb{C}(-b g y+c h z)^{2}=0$, and consequently

$$
\left(g^{2} \mathfrak{C}-h^{2} \mathfrak{A}\right) x-b \sqrt{ } \mathfrak{B}(g \sqrt{ } \mathfrak{C}+h \sqrt{ } \mathfrak{B} \mathfrak{B}) y+c \sqrt{ } \mathfrak{C}(g \sqrt{ } \mathfrak{C}+h \sqrt{ } \mathfrak{B}) z=0:
$$

dividing this by $g \sqrt{ } \mathfrak{C}+h \sqrt{ } \mathbf{B}$, and writing down the system of equations to which the equation thus obtained belongs,

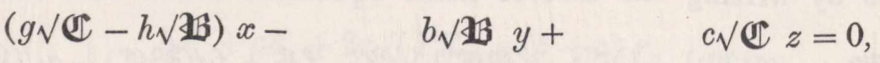

$$
\begin{aligned}
& a \sqrt{ } \mathfrak{A} x+(h \sqrt{ } \mathfrak{A}-f \sqrt{ } \mathfrak{C}) y-\quad c \sqrt{ } \mathfrak{C} z=0, \\
& -a \sqrt{\mathfrak{A}} x+\quad b \sqrt{\mathfrak{B}} y+(f \sqrt{\mathfrak{B}}-g \sqrt{ } \mathfrak{A}) z=0 .
\end{aligned}
$$

C. 
Hence also

$$
\begin{aligned}
& (h \sqrt{\mathfrak{A}}+b \sqrt{\mathfrak{B}}-f \sqrt{ } \mathfrak{C}) y-(\quad g \sqrt{\mathfrak{A}}-f \sqrt{\mathfrak{B}}+c \sqrt{ } \mathfrak{C}) z=0 \\
& (-g \sqrt{\mathfrak{A}}+f \sqrt{\mathfrak{B}}+c \sqrt{ } \mathfrak{C}) z-(a \sqrt{\mathfrak{A}}+h \sqrt{\mathfrak{B}}-g \sqrt{ } \mathfrak{C}) x=0 \\
& (a \sqrt{\mathfrak{A}}-h \sqrt{\mathfrak{B}}+g \sqrt{ } \mathfrak{C}) x-(-h \sqrt{\mathfrak{A}}+b \sqrt{\mathfrak{B}}+f \sqrt{ } \mathfrak{C}) y=0
\end{aligned}
$$

these equations may be written

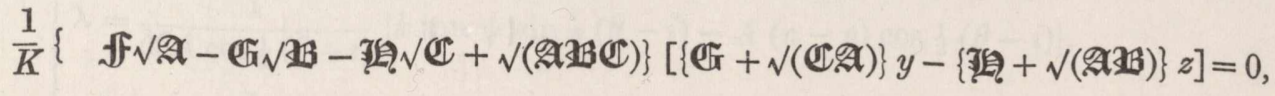

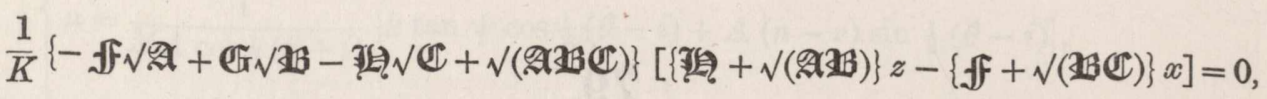

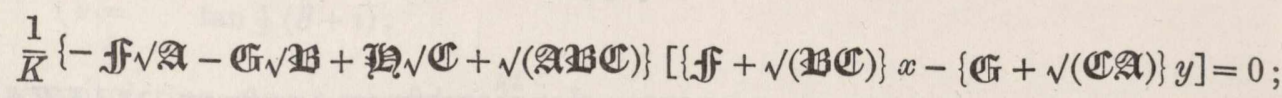

where, as usual,

$$
\begin{aligned}
& \sqrt{\mathfrak{f}}=g h-a f, \quad \mathbb{G}=h f-b g, \quad \text { 私 }=f g-c h, \\
& K=a b c-a f^{2}-b g^{2}-c h^{2}+2 f g h
\end{aligned}
$$

(in fact the coefficient of $y$ in the first equation is

$$
\frac{1}{K}\left\{(\sqrt{\mathfrak{F}} \mathfrak{G}-\mathfrak{C} \mathfrak{T}) \sqrt{\mathfrak{A}}+\left(\mathfrak{A C C}-\mathfrak{G}^{2}\right) \sqrt{\mathfrak{B}}-(\mathfrak{G} \mathfrak{T}-\mathfrak{A} \mathfrak{A}) \sqrt{ } \mathfrak{C}\right\},=h \sqrt{\mathfrak{A}}+b \sqrt{\mathfrak{B}}-f \sqrt{ } \mathfrak{C},
$$

as it should be, and similarly for the coefficients of the remaining terms). We have therefore

$$
\{\mathfrak{f}+\sqrt{ }(\mathfrak{B} \mathfrak{B} C)\} x=\{\mathfrak{G}+\sqrt{ }(\mathfrak{C} \mathfrak{A})\} y=\{\mathfrak{I}
$$

or, what comes to the same thing,

Now

$$
\begin{aligned}
& y z=\frac{1}{2} s\{\sqrt{\mathfrak{F}}+\sqrt{ }(\mathfrak{B C C})\}, \\
& z x=\frac{1}{2} s\{\mathfrak{C}+\sqrt{ }(\mathfrak{C A})\}, \\
& x y=\frac{1}{2} s\{\mathfrak{I}+\sqrt{(\mathfrak{A} \mathfrak{B})}\} .
\end{aligned}
$$

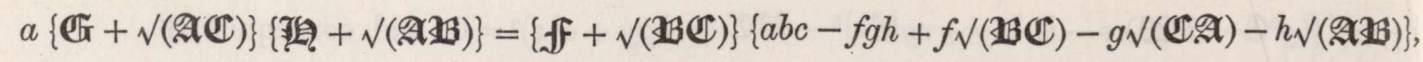

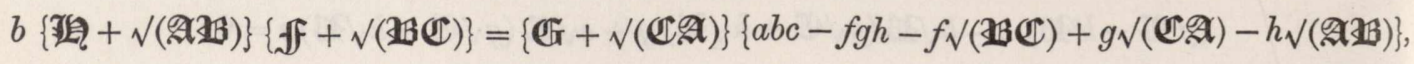

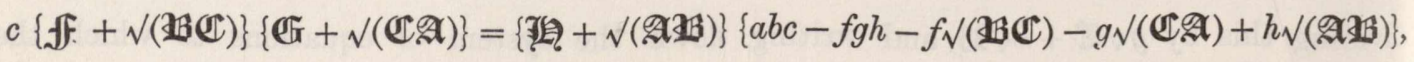
\{as readily appears by writing the first of these equations under the form

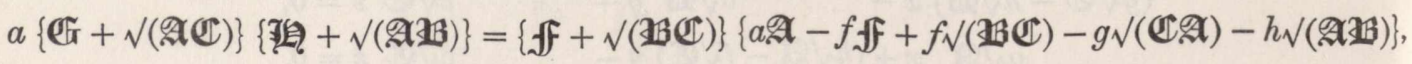
and comparing the rational term and the coefficients of $\sqrt{ }(\boldsymbol{B} \mathfrak{C} \mathfrak{C}), \sqrt{ }(\mathfrak{C} \mathfrak{A}), \sqrt{ }(\mathfrak{A} \mathfrak{A} \mathbf{B})\}$. 
Hence, observing the values of $y z, z x, x y$, we find

$$
\begin{aligned}
& x^{2}=\frac{s}{2 a}\{a b c-f g h+f \sqrt{ }(\mathfrak{B} \mathfrak{C})-g \sqrt{ }(\mathfrak{C} \mathfrak{A})-h \sqrt{ }(\mathfrak{A} \mathfrak{B})\}, \\
& y^{2}=\frac{s}{2 b}\{a b c-f g h-f \sqrt{ }(\mathfrak{B} \mathfrak{C})+g \sqrt{ }(\mathfrak{C A})-h \sqrt{ }(\mathfrak{A} \mathfrak{B})\}, \\
& z^{2}=\frac{s}{2 c}\{a b c-f g h-f \sqrt{ }(\mathfrak{B} \mathfrak{C})-g \sqrt{ }(\mathfrak{C} \mathfrak{A})-h \sqrt{ }(\mathfrak{A} \mathfrak{B})\} .
\end{aligned}
$$

Hence, forming the value of any one of the functions $b y^{2}+c z^{2}+2 f y z, c z^{2}+a x^{2}+2 g x y$, $a x^{2}+b y^{2}+2 h x y$, we obtain $s=\theta^{2}$; or we have

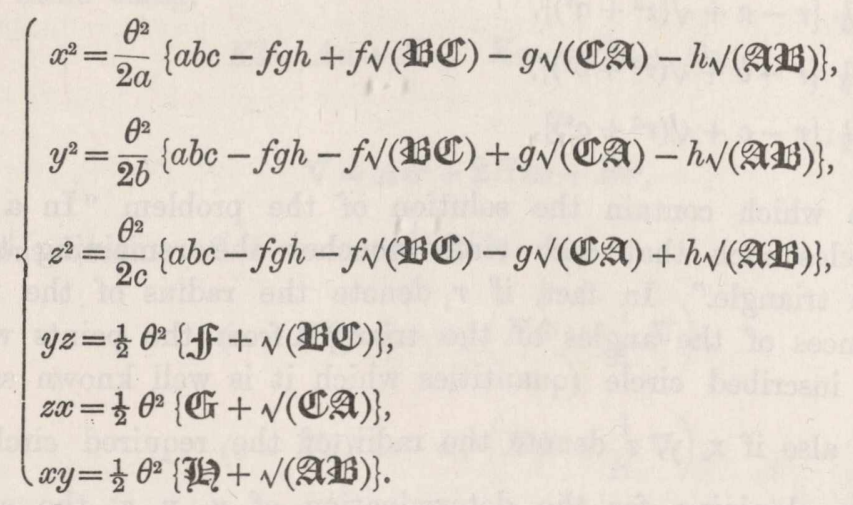

It may be remarked that the equations

$$
\begin{aligned}
& b y^{2}+c z^{2}+2 f y z=L, \\
& c^{\prime} z^{2}+a^{\prime} x^{2}+2 g^{\prime} y z=M, \\
& a^{\prime \prime} x^{2}+b^{\prime \prime} y^{2}+2 h^{\prime \prime} x y=N,
\end{aligned}
$$

in which the coefficients are supposed to be such that the functions

$$
\begin{aligned}
& M\left(a^{\prime \prime} x^{2}+b^{\prime \prime} y^{2}+2 h^{\prime \prime} x y\right)-N\left(c^{\prime} z^{2}+a^{\prime} x^{2}+2 g^{\prime} y z\right), \\
& N\left(b y^{2}+c z^{2}+2 f y z\right)-L\left(a^{\prime \prime} x^{2}+b^{\prime \prime} y^{2}+2 h^{\prime \prime} y z\right), \\
& L\left(c^{\prime} z^{2}+a^{\prime} x^{2}+2 g^{\prime} y z\right)-M\left(b y^{2}+c z^{2}+2 f y z\right),
\end{aligned}
$$

are each of them decomposable into linear factors, may always be reduced to a system of equations similar to those which have just been solved.

Suppose

$$
\left.f=g=h=\frac{1}{\theta^{2}}=\sqrt{\left(\frac{a b c}{a+b+c}\right)}\right)=r,
$$

and write $\sqrt{ } X, \sqrt{ } Y, \sqrt{ } Z$ instead of $x, y, z$. 'The equations to be solved become

$$
\begin{aligned}
& b Y+c Z+2 r \sqrt{ }(Y Z)=(b+c) r \\
& c Z+a X+2 r \sqrt{ }(Z X)=(c+a) r \\
& a X+b Y+2 r \sqrt{ }(X Y)=(a+b) r
\end{aligned}
$$


where $r^{2}=\frac{a b c}{a+b+c}$, and the solution is

$$
\begin{aligned}
\mathrm{x} & =\frac{r}{2 a}\left\{a+b+c-r+\sqrt{ }\left(r^{2}+a^{2}\right)-\sqrt{ }\left(r^{2}+b^{2}\right)-\sqrt{ }\left(r^{2}+c^{2}\right)\right\}, \\
\mathrm{y} & =\frac{r}{2 b}\left\{a+b+c-r+\sqrt{ }\left(r^{2}+a^{2}\right)+\sqrt{ }\left(r^{2}+b^{2}\right)-\sqrt{ }\left(r^{2}+c^{2}\right)\right\}, \\
\mathrm{z} & =\frac{r}{2 c}\left\{a+b+c-r-\sqrt{ }\left(r^{2}+a^{2}\right)-\sqrt{ }\left(r^{2}+b^{2}\right)+\sqrt{ }\left(r^{2}+c^{2}\right)\right\}, \\
\sqrt{ }(\mathrm{yz}) & =\frac{1}{2}\left\{r-a+\sqrt{ }\left(r^{2}+a^{2}\right)\right\}, \\
\sqrt{ }(\mathrm{zx}) & =\frac{1}{2}\left\{r-b+\sqrt{ }\left(r^{2}+b^{2}\right)\right\}, \\
\sqrt{ }(\mathrm{xy}) & =\frac{1}{2}\left\{r-c+\sqrt{ }\left(r^{2}+c^{2}\right)\right\},
\end{aligned}
$$

a system of formulæ which contain the solution of the problem "In a given triangle to inscribe three circles such that each circle touches the remaining two circles and also two sides of the triangle." In fact, if $r$ denote the radius of the inscribed circle, and $a, b, c$ the distances of the angles of the triangle from the points where the sides are touched by the inscribed circle (quantities which it is well known satisfy the condition $\left.r^{2}=\frac{a b c}{a+b+c}\right)$, also if $\mathrm{x}, \mathrm{y}, \mathrm{z}$ denote the radii of the required circles, there is no difficulty whatever in obtaining for the determination of $\mathrm{x}, \mathrm{y}, \mathrm{z}$, the above system of equations. The problem in question was first proposed and solved by an Italian geometer named Malfatti, and has been called after him Malfatti's problem. His solution, dated 1803, and published in the 10th volume of the Transactions of the Italian Academy of Sciences, appears to have consisted in showing that the values first found for the radii of the three circles satisfy the equations given above, without any indication of the process of obtaining the expressions for these radii. Further information as to the history of the problem may be found in the memoir "Das Malfattische Problem neu gelöst von C. Adams," Winterthur, 1846.

In connexion with the preceding investigations may be considered the problem of determining $l$ and $m$ from the equations

$$
\begin{aligned}
& B(l+\theta)^{2}-2 H(l+\theta) m+(A+1) m^{2}=0, \\
& A(m+\theta)^{2}-2 H(m+\theta) l+(B+1) l^{2}=0 ;
\end{aligned}
$$

which express that the function

$$
\theta^{2} U+(l x+m y)^{2}, \quad\left(U=A x^{2}+2 H x y+B y^{2}\right),
$$

has for one of its factors a factor of $U+x^{2}$, and for the other of its factors a factor of $U+y^{2}$. There is no difficulty in solving these equations; and if we write

$$
K=A B-H^{2}, \quad \varpi_{1}=\sqrt{ }(-K-B), \quad \varpi_{2}=\sqrt{ }(-K-A),
$$


the result is easily shown to be

$$
l: m: \theta=A\left(B+H+\varpi_{1}\right): B\left(A+H+\varpi_{2}\right):\left(H+\varpi_{1}\right)\left(H+\varpi_{2}\right)-A B .
$$

But the problem may be considered as the problem for two variables, analogous to that of determining the conic having a double contact with a given conic, and touching three conics each of them having a double contact with the given conic; and in this point of view I was led to the following solution. If we assume

$$
B l-H m=u, \quad-H l+A m=v,
$$

or, what is the same thing,

$$
K l=A u+H v, \quad K m=H u+B v,
$$

then putting

$$
\nabla=A u^{2}+2 H u v+B v^{2},
$$

the two equations become after some reduction

$$
\begin{aligned}
& (u-K \theta)^{2}=-\varpi_{1}^{2}\left(K \theta^{2}+\frac{1}{K} \nabla\right), \\
& (v-K \theta)^{2}=-\varpi_{2}^{2}\left(K \theta^{2}+\frac{1}{K} \nabla\right) .
\end{aligned}
$$

Hence, writing $K \theta^{2}+\frac{1}{K} \nabla=-s^{2}$, we have

$$
u=K \theta+\varpi_{1} s, \quad v=K \theta+\varpi_{2} s, \quad \nabla+K^{2} \theta^{2}+K s^{2}=0 ;
$$

and substituting these values of $u, v$ in the last equation,

$$
A\left(K \theta+\varpi_{1} s\right)^{2}+2 H\left(K \theta+\varpi_{1} s\right)\left(K \theta+\varpi_{2} s\right)+B\left(K \theta+\varpi_{2} s\right)^{2}+K^{2} \theta^{2}+K s^{2}=0,
$$

or reducing,

$K^{2} \theta^{2}(A+2 H+B+1)+2 K \theta s\left\{(A+H) \varpi_{1}+(H+B) \varpi_{2}\right\}+s^{2}\left(A \varpi_{1}{ }^{2}+2 H \varpi_{1} \varpi_{2}+B \varpi_{2}{ }^{2}+K\right)=0 ;$

whence

$$
\begin{aligned}
& {\left[K \theta(A+2 H+B+1)+s\left\{(A+H) \varpi_{1}+(H+B) \varpi_{2}\right\}\right]^{2}} \\
& =s^{2}\left[\left\{(A+H) \varpi_{1}+(H+B) \varpi_{2}\right\}^{2}-(A+2 H+B+1)\left(A \varpi_{1}^{2}+2 H \varpi_{1} \varpi_{2}+B \varpi_{2}{ }^{2}+K\right)\right], \\
& =s^{2}\left\{-K\left(\varpi_{1}-\varpi_{2}\right)^{2}-\left(A \varpi_{1}{ }^{2}+2 H \varpi_{1} \varpi_{2}+B \varpi_{2}^{2}\right)-K(A+2 H+B+1)\right\}, \\
& =s^{2}\left\{-(A+K) \varpi_{1}^{2}-(B+K) \varpi_{2}^{2}-K(A+2 H+B+1)+2(K-H) \varpi_{1} \varpi_{2}\right\}, \\
& =s^{2}\left\{2 \varpi_{1}^{2} \varpi_{2}^{2}-K(A+2 H+B+1)+2(K-H) \varpi_{1} \varpi_{2}\right\}, \\
& =s^{2}\left(\varpi_{1} \varpi_{2}+K-H\right)^{2}:
\end{aligned}
$$


and therefore

$$
K \theta(A+2 H+B+1)+s\left\{(A+H) \varpi_{1}+(H+B) \varpi_{2}\right\}=s\left(\varpi_{1} \varpi_{2}+K-H\right),
$$

giving

$$
s=\frac{K \theta(A+2 H+B+1)}{\varpi_{1} \varpi_{2}-(A+H) \varpi_{1}-(H+B) \varpi_{2}+K-H} .
$$

But

$$
K l=A u+H v=(A+H) K \theta+\left(A \varpi_{1}+H \varpi_{2}\right) s, \quad K m=(H+B) K \theta+\left(H \varpi_{1}+B \varpi_{2}\right) s,
$$

and substituting the above value of $s$, we obtain, after some simple reductions,

$$
\begin{aligned}
l: m: \theta=\left(\varpi_{1} \varpi_{2}+K-H\right)\left(A+H-\varpi_{2}\right):\left(\varpi_{1} \varpi_{2}+K-H\right)\left(B+H-\varpi_{2}\right) \\
:\left\{\varpi_{1} \varpi_{2}-(A+H) \varpi_{1}-(B+H) \varpi_{2}+K-H\right\},
\end{aligned}
$$

a result which presents itself in a very different form from the one previously obtained: if, however, the terms of: this proportion be multiplied by the factor

$$
\frac{H \varpi_{1} \varpi_{2}-K \varpi_{1}-K \varpi_{2}+A B-K H}{(A+2 H+B+1) K},
$$

they become (as they ought to do) identical with those of the former proportion, and the identical equations to which this process gives rise are not without interest. 